\title{
Geospheric Effects of Magnetic Clouds during 1996-2008
}

\author{
S Kumar' ${ }^{1}$, R Verma ${ }^{2}$, S K Dubey ${ }^{3}$ \\ ${ }^{1}$ H.No.992/3036, Jabali Dant Chikitsalaya, Survey of India, Behind SBI Qrts., Vijay Nagar, Jabalpur (M.P.) - 482001. India \\ ${ }^{2}$ S.G.T.B.Khalsa College, Jabalpur (M.P.) - 482001. India \\ ${ }^{3}$ Auto. Govt. Science College, Jabalpur (M.P.) - 482001. India
}

\begin{abstract}
The purpose of this analysis is to investigate the different characteristics of Magnetic Clouds (MCs) which have occurred during Solar cycle 23 and in the ascending phase of Solar cycle 24 and to study their effect on geomagnetosphere so as to have better understanding of the association of GMSs with MCs. Ninety eight MCs are observed and examine during the period of 1996 to 2008. The dependence of geomagnetic activity on solar features and interplanetary (IP) parameters is investigated. Ninety eight weak ( $-30 \mathrm{nT}$ $>$ Dst $\geq-50 n T)$, moderate ( $-50 n T>D s t \geq-100 n T)$, intense with $(-200 n T \leq D s t<-100 n T)$ and superintense (Dst $<-200 n T)$ geomagnetic stroms ( GMSs) have been studied from 1996 to 2008. The collected sample events are divided into two groups based on their association with MCs related to geomagnetic storms Dst $\leq-50 \mathrm{nT}$ eg. 1. geoeffective events and 2. For nongeoeffective events Dst $\geq$ $-50 n T$. The number of GMSs show three distinct peaks during the 11-year period of $23^{\text {rd }}$ solar cycle. Solar and IP parameters, e.g., Vsw, $B z$ and their products, e.g., Vsw.Bz, are observed and correlated to predict the occurrence of GMSs. The correlation coefficient, $r=-0.60$, 0.15 and 0.21 of parameters $V s w, B z$ and $V s w . B z$ respectively, with Dst. The correlation coefficient for intense and superintense GMSs, $r$ $=-0.64,0.30$ and 0.31 of parameters $V s w, B z$ and $V s w . B z$ respectively, with Dst.
\end{abstract}

Keywords: Magnetic Cloud; Solar Wind; Geomagnetic Storms

PACS Nos. 96.60.ph; 96.50.Uv; 96.50.Ci

\section{Introduction}

In recent years a number of investigations have been carried out to understand the solar terrestrial relationship and to ascertain factors that are responsible for $\operatorname{GMSs}(1,2)$. It is believed that the GMSs are the response to interplanetary (IP) phenomena arising as a consequence of a solar event. The geospheric environment is highly affected by the Sun and its features such as Solar Flares (SFs), Active Prominences and Disappearing Filaments (APDFs), Coronal Holes (CHs), CMEs etc. Research since last three decades identifies CMEs as the energetic events in the heliosphere. CMEs from the Sun drive, Solar Wind (SW) disturbances in terms of magnetic field, speed and density which in turn cause geomagnetic disturbances at Earth.

The term Magnetic cloud is originally used by Parker in 1957 in a much broader sense in his theoretical study of the dynamics of hydromagnetic gas clouds ejected from the Sun into the IP space (3). Further, it is used by Burlaga et al. (1981) who defined MCs as a region of enhanced magnetic field strength, smooth rotation of the magnetic field vector and low proton temperature (4). CMEs that are observed in the solar wind near 1 AU are commonly called interplanetary coronal mass ejection (ICMEs). $\mathrm{MC}$ is the subset of ICMEs having a specific configuration in which the magnetic strength is higher than the average magnetic field. A MC is a transient event observed in the solar wind. MCs are a possible manifestation of CMEs. MCs are often but not always associated with IP shocks. MCs that strike the Earth may cause intense GMSs; Bothmer and schwenn, 1998 (5).

\section{Data Analysis}

During the period 1996 -2008 Shocks associated with magnetic clouds have been investigated. The data on Magnetic Clouds is obtained from MFI table of MAG CLOUD'S (File://G:IMFI TABLE OF MAG CLOUD'S.htm) covering the above period and containing 98 events. The values of Dst indices are taken from world data center, Japan (http://swdcwww.kugi.kyoto-u.ac.jp). The data on Shocks is obtained from ACE Lists of Disturbances and Transients. Geomagnetic response of MCs has been studied on the basis of Disturbance storm time (Dst) index represents the deviation in the horizontal component of the Earth's magnetic field at the equator relative to its value in a quiet day. In the present investigation, the GMSs have been classified as : weak ( $-30 \mathrm{nT}>\mathrm{Dst} \geq-50 \mathrm{nT})$, moderate $(-50$ $\mathrm{nT}>$ Dst $\geq-100 \mathrm{nT})$ and intense with Dst $<-100 \mathrm{nT}$ and superintense ( Dst $<-200 \mathrm{nT}$ )

During the passage of MCs, both northward and southward magnetic fields are observed. However, southward field is crucial for the reconnection process. This has been established by now that GMSs occur when the southward component of Interplanetary Magnetic Field (IMF), Bz impinges upon the Earth's magnetosphere and reconnect. Ninety eight Magnetic clouds (MCs) are observed and analysed during the period from 1996 to 2008 .

\section{Results and Discussion}

In the present study, MCs are classified into two major categories, as unipolar and bipolar depending upon the change in magnetic field direction between arrival and departure of MC as observed at $1 \mathrm{AU}$. Unipolar MCs have 


\section{International Journal of Science and Research (IJSR) \\ ISSN (Online): 2319-7064}

Index Copernicus Value (2013): 6.14 | Impact Factor (2014): 5.611

either southward $(\mathrm{S})$ or northward $(\mathrm{N})$ magnetic field for most of the time of MC's duration at 1 AU which almost similar to Gopalswamy et al. 2008 (1). Bipolar MCs have magnetic field either changing from southward to northward $(\mathrm{SN})$ or vice versa (NS).

The entire period under consideration, is divided into three parts: Rising phase from 1996 to 1998 and 2007 to 2008, maximum phase from 1999 to 2002 and declining phase from 2003 to 2006. During the year 1999, only four MCs are observed which is sometime unexpected since the year 1999 is close to the maximum phase; whereas, in 1997 which is in the rising phase and close to minimum phase, largest number of MCs of $23^{\text {rd }}$ solar cycle are observed. MCs do not reveal any systematic relationship with Solar activity, apart from the fact that on both the minimum years i.e. during 1996 and 2006 , the number of MCs is very less. During Solar cycle $23,36 \%$ MCs are observed to be unipolar while $64 \%$ are bipolar. $\mathrm{S}$ or $\mathrm{N}$ type unipolar MCs are found to be equal in number during the Solar cycle 23, whereas, SN type of bipolar MCs are more dominating than NS type with their pretty high abundance of $61 \%$ which is similar to the observations of Zhang and Burlaga, 1988 (6), who stated that $\mathrm{SN}$ rotation of magnetic field vector is more frequent. Out of the total MCs, $62 \% \mathrm{MCs}$ are observed to be geoeffective and $79 \%$ of them are associated with shock. Majority of non geoeffective MCs are observed during rising phase.

It is observable that $20 \%, 25 \%, 28 \%$ and $9 \%$ MCs lead to weak, moderate, intense and superintense GMSs respectively. Majority of the intense (78\%) GMSs have occurred during maximum and declining phases of Solar activity; whereas majority of the weak (53\%) GMSs are observed during rising phase of Solar activity. However, moderate GMSs are more or less equally distributed among all the three phases of Solar activity.

More than half of weak (53\%), moderate $(61 \%)$ and intense $(60 \%)$ GMSs are caused due to SN and S type MCs, which implies that SN and S type clouds are comparatively more geoeffective. Further, $83 \% \mathrm{~N}$ type MCs lead to GMSs which could be due to the presence of southward component in the sheath region. The number of MCs having high speed are less. Maximum MCs have speed in the range 300-500 Km/s. The average speed of MCs is observed to be $475.6 \mathrm{Km} / \mathrm{s}$.

\section{Conclusions}

Based on the present investigations of the characteristics of the MCs observed and their geomagnetic consequences, the following conclusions have been derived:

1) $\mathrm{SN}$ type of MCs dominates over NS type of MCs during Solar cycle 23. However, $\mathrm{S}$ and $\mathrm{N}$ type unipolar MCs are found to be almost equal in number.
2) $87 \%$ of the total MCs observed during Solar cycle 23 are found to be geoeffective. Majority of the MCs lead to intense GMSs; whereas, highly intense GMSs of Dst $<-300 \mathrm{nT}$ follow from SN or S type MCs.

3) $\mathrm{SN}$ and $\mathrm{S}$ type clouds are found to be more geoeffective. Some of the $\mathrm{N}$ type MCs also lead to GMSs which cloud be due to the presence of southward $\mathrm{Bz}$ component in sheath region.

4) No preference is observed for RH or LH clouds for being geoeffective.

5) Speed of MCs seems to be one of the important parameter responsible for the occurrence of GMS. Geoeffective MCs generally have high speed. MCs of very high speed lead to intense GMSs. Speed of the MC appears to have greater impact on the minimum value of Dst attained during a GMS.

6) It is not always true that $\mathrm{SN}$ clouds lead to more fall in Dst value than NS clouds for similar value of $\mathrm{Bz}$ minimum associated with both type of MCs.

7) Only four MCs are observed in 2007, that one without shocks. One of these MCs lead to GMS of moderate nature.

8) The magnetic clouds have velocity greater than 400 $\mathrm{Km} / \mathrm{s}$ are mostly responsible for GMSs.

9) Magnetic cloud velocity has significant anti-correlation with the Dst and it is also one of the important parameter for prediction of the GMSs.

10) Duration of encounter of MCs at $1 \mathrm{AU}$ has no effect on GMSs.

\section{Acknowledgements}

The authors are highly indebted to various experimental groups for providing data online through Internet. They are also grateful to Prof. N. Gopalswamy and other authors for providing valuable information on MCs and GMSs directly or indirectly. The authors acknowledge the contributions from several of his colleagues.

\section{References}

[1] Gopalswamy N, Akiyama S, Yashiro S, Michalek G and Lepping R P 2008 J. Atmos. Sol. Terr. Phys. Vol. 70245.

[2] Kumar S and Raizada A 2008 Pramana Journal of Physics Vol.71 1353.

[3] Parker E N 1957 Astrophys. J. Supp. Vol. 351.

[4] Burlaga L F, Sitter E, Mariani F and Schwenn R 1981 J. Geophys. Res. Vol. 866673.

[5] Bothmer V and Schwenn R 1998 Ann. Geophys. Vol. 16 1.

[6] Zhang G and Burlaga L F 1988 J. Geophys. Res. Vol. 93 2511. 
International Journal of Science and Research (IJSR)

ISSN (Online): 2319-7064

Index Copernicus Value (2013): 6.14 | Impact Factor (2014): 5.611

RDVV, Jabalpur

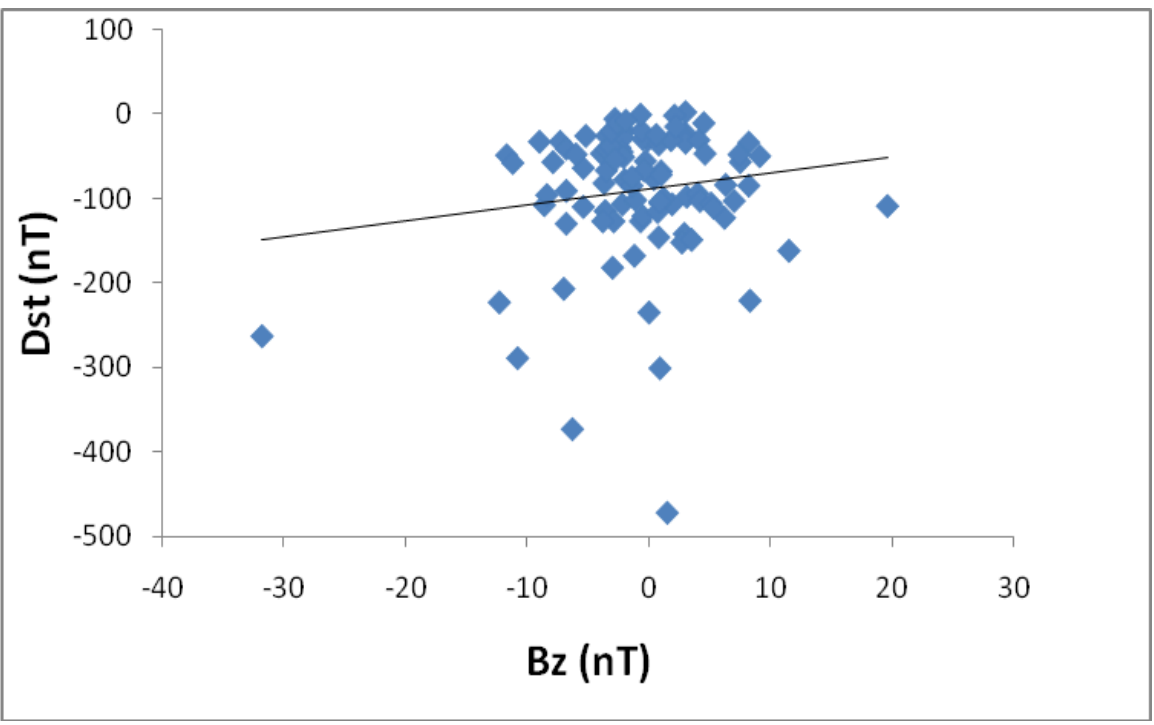

Figure 1: The scatter plot between Bz and Dst have been plotted

RDVV, Jabalpur

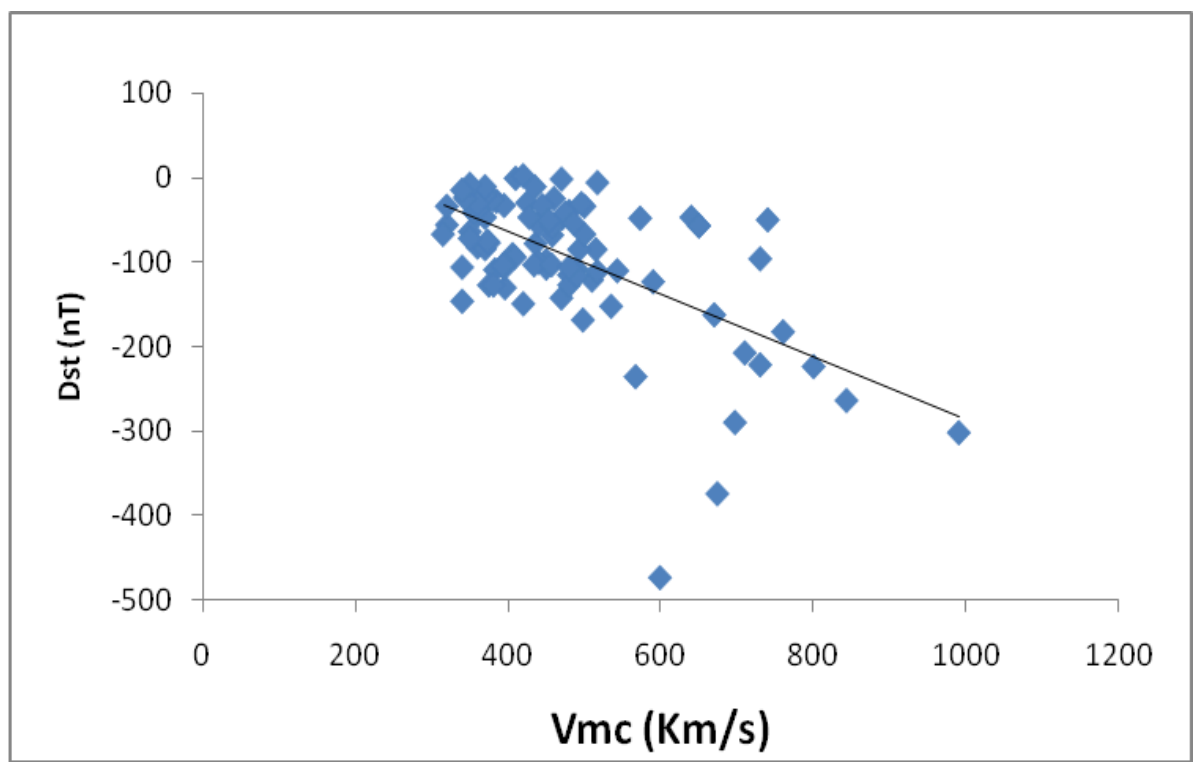

Figure 2: The scatter plot between Vmcs Dst have been plotted

RDVV, Jabalpur

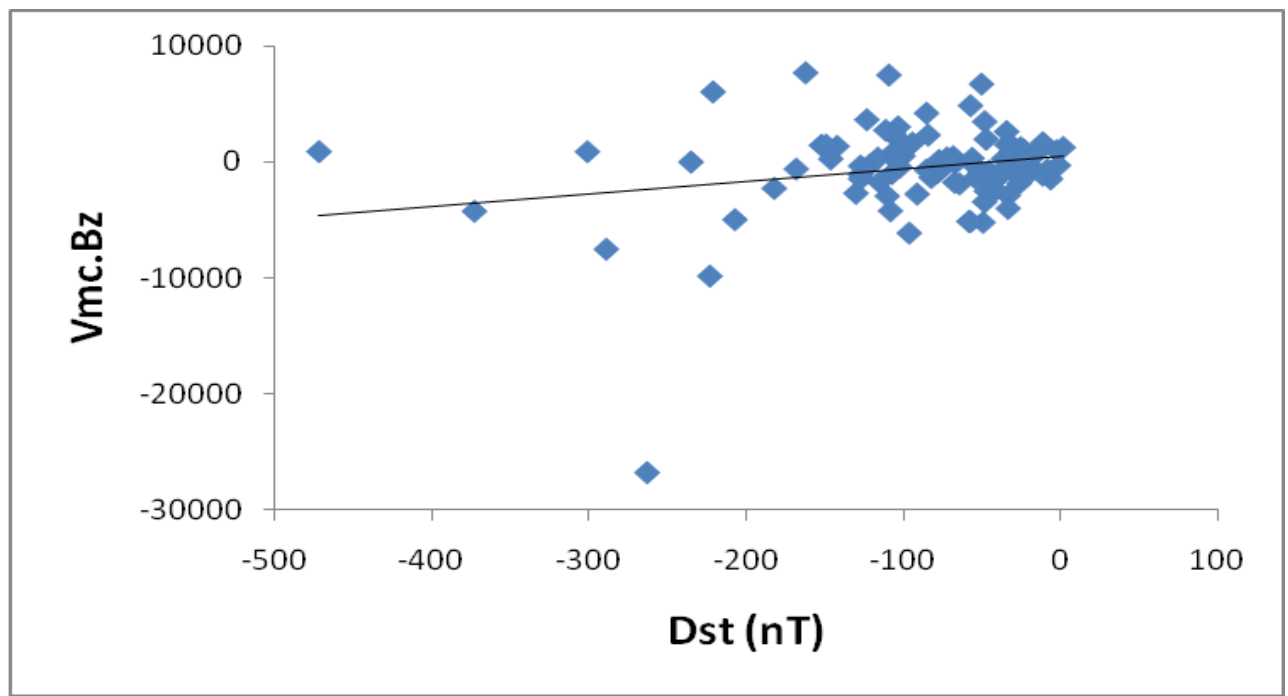

Figure 3: The scatter plot between Dst and $V_{m c}$.Bz have been plotted

Volume 5 Issue 1, January 2016

www.ijsr.net 
RDVV, Jabalpur

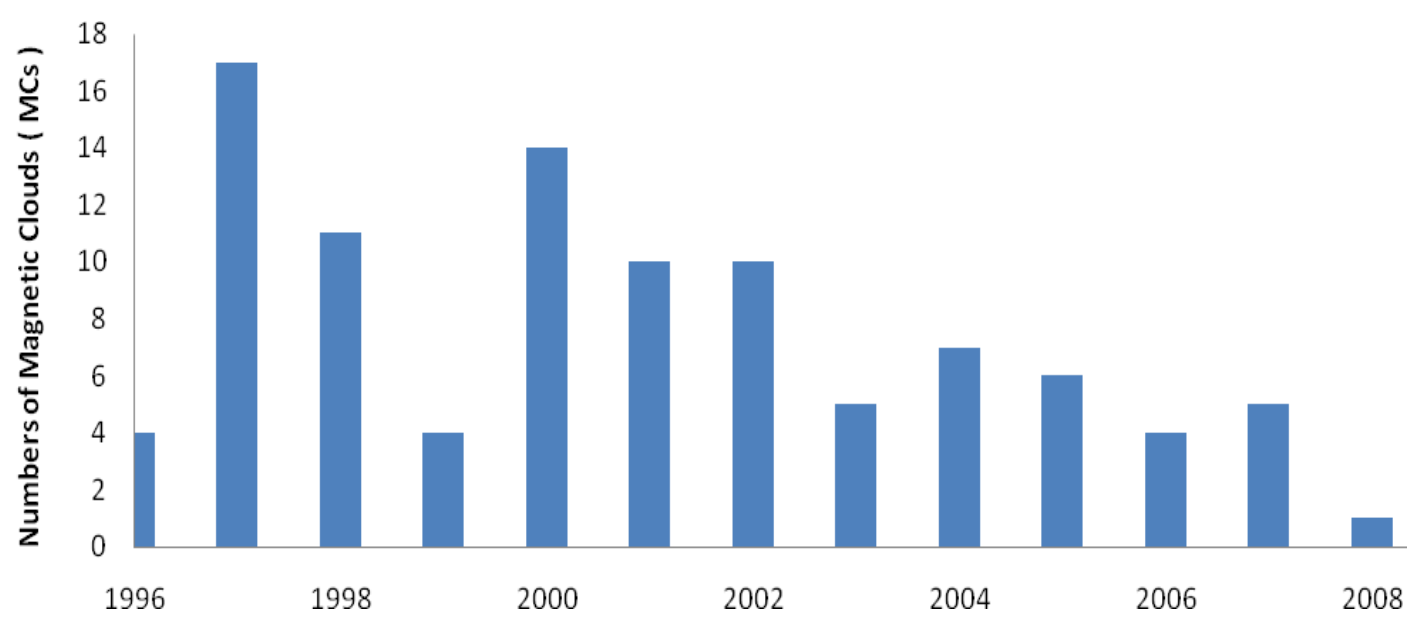

Figure 4: Yearly Distribution of MCs has been plotted histographically 